Gerard W.K. Hugenholtz · Joost J. Stolker

Eibert R. Heerdink · Willem A. Nolen

Hubert G.M. Leufkens

\title{
Short-acting parenteral antipsychotics drive choice for classical versus atypical agents
}

Received: 29 June 2002/ Accepted: 20 November 2002/Published online: 13 February 2003

(C) Springer-Verlag 2003

\begin{abstract}
Objective: The objective of this study was to investigate which antipsychotics (classical versus atypical) are prescribed in a psychiatric hospital and which determinants affect the choice for one of these two classes of antipsychotics in newly admitted patients. Methods: In a retrospective cohort design, 522 newly admitted patients were followed from the date of admission until discharge from the hospital. In the cohort of newly admitted patients treated with an oral antipsychotic, a nested case-control study was conducted considering recipients of an atypical agent as cases. Controls were all other cohort members. The association of patient characteristics and the choice between classical versus atypical antipsychotics was studied using logistic regression analysis. The same analysis was performed with adjustment for possible confounding factors (age group, gender, DSM-IV diagnoses, use of shortacting parenteral antipsychotic, global assessment of functioning score, involuntary admissions and involuntary measures).

Results: Patients treated with classical oral antipsychotics were more often previously treated with short-acting parenteral antipsychotics than patients treated with atypical antipsychotics $(40.8 \%$ vs $15.2 \%$; adjusted $\mathrm{OR}=0.2095 \% \mathrm{CI}=0.09-0.44)$. No statistically significant difference was found between patients with different severities of disease.
\end{abstract}

G.W.K. Hugenholtz $(\bowtie) \cdot$ J.J. Stolker $\cdot$ W.A. Nolen

Altrecht Institute for Mental Health Care,

Utrecht, The Netherlands

E-mail: g.hugenholtz@altrecht.nl

Tel.: + 31-30-2256556

Fax: + 31-30-2256987

G.W.K. Hugenholtz · J.J. Stolker · E.R. Heerdink

H.G.M. Leufkens

Department of Pharmacoepidemiology and Pharmacotherapy,

Utrecht Institute for Pharmaceutical Sciences (UIPS),

PO Box 80082, 3508 TB Utrecht, The Netherlands

W.A. Nolen

Department of Psychiatry,

University Medical Centre Utrecht, Utrecht, The Netherlands
Discussion: Availability of injectable forms seems to drive the choice for oral antipsychotic agents. Future introductions of short-acting parenteral atypical antipsychotics may have a large impact on first-choice oral antipsychotic treatment.

Keywords Antipsychotics - Parenteral determinant · Epidemiology

\section{Introduction}

Antipsychotics are essential in the treatment of patients suffering from psychotic disorders, in clinical as well as in community settings [1]. Classical (typical) antipsychotic drugs such as haloperidol, pimozide and others are widely used as first-choice treatments for acute and chronic psychotic disorders [2]. However, these substances have a relatively limited effect on negative symptoms associated with schizophrenia, i.e. poverty of speech, lack of motivation, apathy and inability to express emotions [3]. Moreover, their use is associated with disabling adverse effects, including extrapyramidal symptoms (EPS) [4, 5].

The introduction of atypical antipsychotics (clozapine, olanzapine, risperidone, quetiapine and ziprasidone) has changed treatment options for psychotic disorders. There has been a large shift in favour of the use of these atypical antipsychotics [6], although the precise therapeutic value of these agents remains controversial $[7,8,9,10,11,12,13]$. The effect on negative symptoms has not yet been fully elucidated. All the newer agents seem to be superior with regard to risk on EPS but they have been associated with other side effects, such as weight gain [14]. Official therapeutic guidelines, including the one on the pharmacotherapy of psychotic disorders in The Netherlands [15], had not yet decided between classical or atypical antipsychotics as first-choice treatment. For insight into prescribing patterns of antipsychotics in daily practice and into factors 
that affect the choice between classical and atypical antipsychotics, an observational study within a welldefined group is needed.

The objective of this study was to investigate which groups of antipsychotics (classical versus atypical) are prescribed to newly admitted patients in a psychiatric hospital, and which determinants affect the choice for one or the other class of antipsychotics.

\section{Methods}

Setting and study population

Data were retrospectively collected from the acute psychiatric admission wards of three psychiatric hospitals, serving a catchment area of about 720,000 inhabitants in the centre of The Netherlands, during the period 1997-1999. Patients, aged between 18 years and 60 years old, who were admitted for a new hospitalisation of at least 3 days were included in the cohort. 'Newly hospitalised' was defined as having no previous admission to the psychiatric centre for any indication in the 2 years before the inclusion date. We collected data back until 1995 for the 1997 admissions.

\section{Design}

In a retrospective cohort design, patients were followed from date of admission until discharge from the hospital. In the cohort of newly admitted patients treated with an oral antipsychotic, a nested case control study was conducted considering recipients of an atypical agent as cases. Controls were all other cohort members.

The drug use database and the clinical database were linked anonymously through record linkage methodology based on date of birth, gender and day of admission [16]. At admission, diagnoses were coded according to criteria based on the Diagnostic and Statistical Manual of Mental Disorders, (DSM)-IV [17] by the treating psychiatrists. Patients were rated on the "Global Assessment of Functioning" score (GAF). A low GAF score is a measure of more severe illness. The admissions were classified as "voluntary" or "involuntary". Involuntary measures at admission were also classified. Involuntary admissions and involuntary measures are reserved for more severely ill patients.

Antipsychotics were classified as classical or atypical. Clozapine, olanzapine, risperidone, sertindole and quetiapine were classified as atypical antipsychotics. Other drugs with four digits ATC-code N05A[18] were classified as classical antipsychotics. Lithium and levomepromazine also have a four-digits ATC-code N05A but were excluded because they are not registered for psychotic disorders in the Netherlands.

Drugs were stratified according to their route of administration: oral or parenteral. We differentiated between parenteral shortacting (e.g. haloperidol and zuclopenthixol-acetate) and long-acting $(=$ depot $)$ antipsychotics. We excluded 31 patients who received both an oral classical and an oral atypical antipsychotic on the day of admission. Patients receiving depot antipsychotics were excluded because atypical antipsychotic medication was not available, so a choice between classical and atypical was not possible.

The Scientific Committee and the board of the centre for mental health approved the study protocol with respect to privacy aspects.

\section{Analysis}

The association of patient characteristics and the choice between classical versus atypical antipsychotics was studied using logistic regression analysis. Associations were estimated as odds ratios with corresponding $95 \%$ confidence interval $(95 \% \mathrm{CI})$. The same analysis was performed with adjustment for possible confounding factors (age group, gender, DSM-IV diagnoses, use of short-acting parenteral antipsychotic, GAF score, involuntary admissions and involuntary measures). Data were analysed using EGRET statistical software (version 2.0.31) by Cytel Software Corporation.

\section{Results}

The characteristics of the cohort members are presented in Table 1. A total of 522 patients met the inclusion criteria. Most patients $(60.9 \%)$ were younger than 40 years old, with a median age of 36 years. Psychotic disorders accounted for $50.2 \%$ of the diagnoses of the patients admitted. Other diagnoses included bipolar disorders $(16.3 \%)$, depressive disorders $(12.6 \%)$ and personality disorders $(16.7 \%)$. The most frequently prescribed oral antipsychotic drugs were zuclopenthixol (33.7\%), pimozide (13.4\%) and haloperidol (12.6\%). The proportion of atypical agents was $27.8 \%$, consisting of clozapine $(1.9 \%)$, olanzapine $(14.8 \%)$ and risperidone $(11.1 \%)$.

In Table 2, possible determinants of type of first oral antipsychotic used, are listed. We found 154 (40.8\%) of 377 patients treated with classical oral antipsychotics, which were initially treated with short-acting parenteral antipsychotics. This was $15.2 \%$ in the group treated with an oral atypical antipsychotic (adjusted OR $=0.2095 \%$ $\mathrm{CI}=0.09-0.44)$. The use of atypical antipsychotics was significantly lower in patients with bipolar disorders (adjusted $\mathrm{OR}=0.3695 \% \mathrm{CI}=0.13-0.95$ ).

No statistically significant difference was found between patients with different severities of disease at the time of admission, indicated by GAF score, involuntary admission and involuntary measures. GAF scores were missing in $180(34.4 \%)$ of the patients. Analysis of these missing GAF scores revealed that most missing GAF scores were from the 1997 admissions, where $113(64.6 \%)$ GAF scores of 175 admissions were missing; in 1998, there were $26(13.6 \%)$ of 191 and $41(26.3 \%)$ of 156 .

\section{Discussion}

This study showed that availability of injectable forms seems to drive the choice for oral antipsychotic agents in patients newly admitted to a psychiatric hospital. Five

Table 1 Characteristics of the newlyadmitted patients

\begin{tabular}{ll}
\hline Age (years) & $n=522$ \\
$<40$ & $318(60.9 \%)$ \\
$40-60$ & $204(39.1 \%)$ \\
Median age (years) & 36 \\
Female gender & $249(47.7 \%)$ \\
Any diagnosis (DSM-IV)* & \\
Psychoticdisorders & $262(50.2 \%)$ \\
Bipolar disorders & $85(16.3 \%)$ \\
Depressive disorders & $66(12.6 \%)$ \\
Personality disorders & $87(16.7 \%)$ \\
Anxiety disorders & $26(5.0 \%)$ \\
Other disorders & $23(4.4 \%)$ \\
Unknown disorders & $65(12.5 \%)$ \\
\hline
\end{tabular}

*Totals exceed $100 \%$ because of multiple diagnoses 
Table 2 Initial oral antipsychoticuse of newly admitted patients

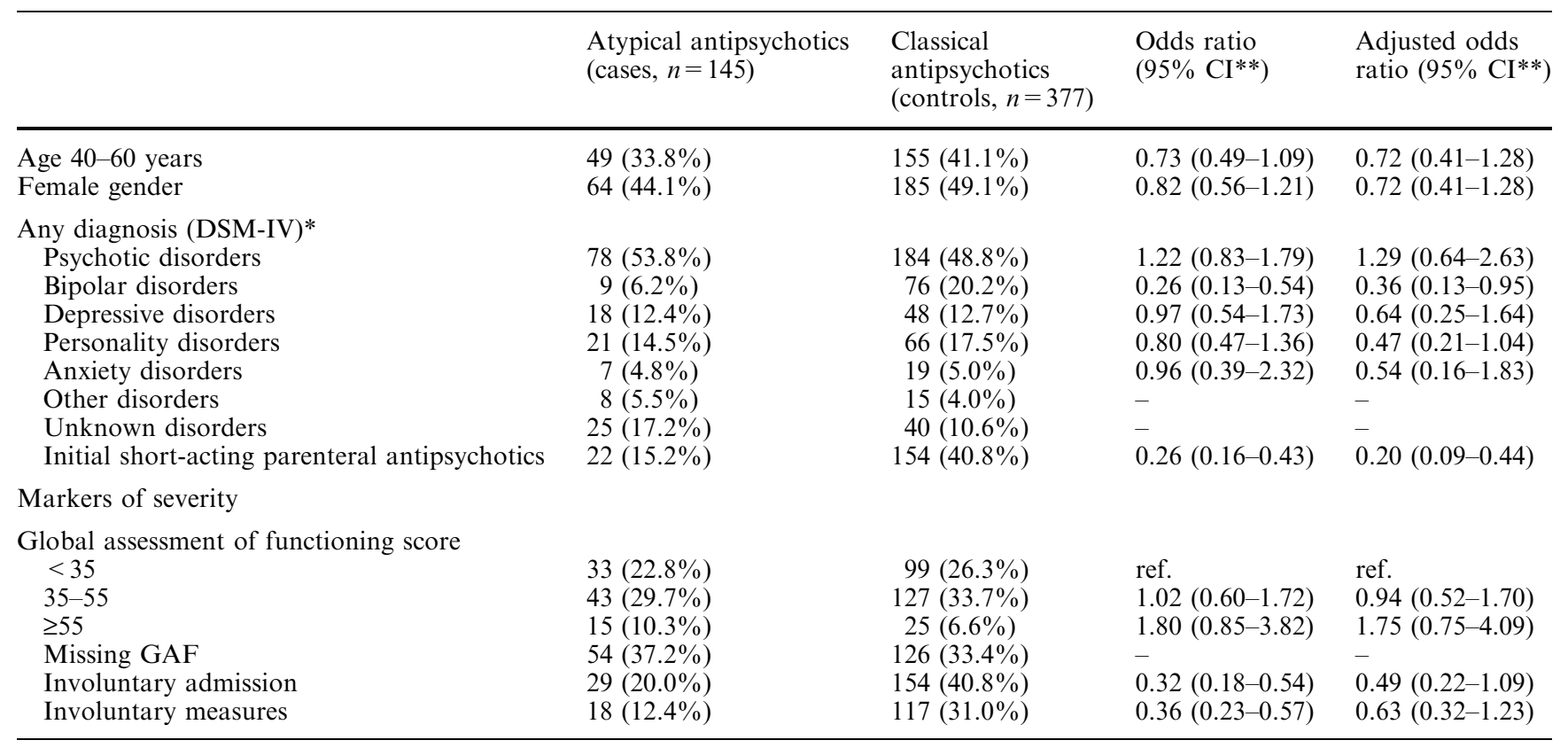

*Totals exceed $100 \%$ because of multiple diagnoses

$* * 95 \%$ Confidence interval

times as many patients treated with oral classical antipsychotics than oral atypical antipsychotics were initially treated with short-acting parenteral agents.

Patients with psychotic illnesses may have delusions or hallucinations that may lead them to be aggressive or violent to themselves or others. Medication that is used in this context requires the properties of rapid onset of effect (tranquillisation or at least initial sedation in order to control aggressive or disorganised behaviour) [19]. Antipsychotic effect is also needed, but cannot be expected within one or two weeks [20]. In this context, it is an unexpected finding that the markers of severity (GAF scores, involuntary admissions and involuntary measures) of the patients receiving oral atypical antipsychotics did not differ from patients treated with oral classical antipsychotics. Although no statistical significance was found, it may be the case that severity of the disease is a determinant of the choice between patients in both groups.

In additional analyses, we included more variables (e.g. marital status, living situation before and after admission etc.) but found no association with choice of antipsychotic. In the final analysis, we included only those variables for which an association could be expected, based on prior publication.

After admission to a ward for acute psychiatric disorders, many patients with psychotic disorders were initially treated with a parenteral antipsychotic. The choice for one of the available injectable forms with immediate action, frequently done in a situation when rapid response to a psychotic crisis is needed, also affects follow-up treatment scenarios, assuming the administration of an antipsychotic results in a positive effect (e.g. control of aggression) on the acute status of the patient. As a result, the physician will often choose to continue the same type of medication in an oral formulation. The choice of oral medication seems to express the satisfaction about the effect of the short-acting parenteral antipsychotic on the non-psychotic symptoms. Moreover, in patients who have received a shortacting parenteral classical antipsychotic without troublesome adverse effects are more likely to continue their classical antipsychotic therapy.

At the time of the study, the official Dutch guidelines for prescribing antipsychotics in schizophrenic psychosis [15] had not yet decided between classical or atypical antipsychotics as first-choice treatment. In the three hospitals, no financial or administrative barriers were made to prevent physicians from prescribing new and expensive atypical antipsychotics.

The large difference between initial use of classical and atypical antipsychotics is in line with the official Dutch guidelines for pharmacotherapy in bipolar disorders [21]. When antipsychotics are needed, the guidelines suggest classical antipsychotics or clozapine for these disorders.

There are some limitations to our study. One can argue that only data on admitted patients were available. However, we were interested in the more severely ill patients, who were admitted to a psychiatric hospital. Another limitation is the possibility that our selection of newly admitted patients may contain some patients previously admitted in another region, before moving to the catchment area of our hospital. Since patients in the Netherlands are preferably transferred to their home region, this will consist of a minority of the included patients.

Although we collected data from only three hospitals, the catchment area these hospitals serve is a rather large 
area, where admission in most cases will lead to admission to one of the investigated hospitals. However, it is a limited geographical area, and therefore it can be argued that the results are not representative for other regions in the Netherlands or elsewhere.

Unfortunately, more than $30 \%$ of GAF scores were missing. Most missing GAF scores, however, were connected to the 1997 admissions. During this period, it was not yet common practice to fill in GAF scores into the hospital database. In 1998 and 1999 more attention was given to this subject. Moreover, patients with available GAF scores did not differ in gender, age and only slightly in diagnostic categories when compared with patients with missing GAF scores. It is therefore likely that our data are representative of the total population of patients.

If clinicians prescribe parenteral antipsychotics, there is no other choice than to prescribe classical antipsychotics at this time, since atypical antipsychotics are not yet available in parenteral formulations. Our study reveals that initial use of short-acting parenteral antipsychotics is also-after adjusting for possible confounding factors-a major determinant for the first-choice oral antipsychotic treatment. Therefore, we anticipate that upcoming introductions of short-acting parenteral atypical antipsychotics may have a large impact on firstchoice oral antipsychotic treatment. Because of much higher pricing of atypical antipsychotics, a further shift in favour of the atypical antipsychotics will have a large impact on hospital budget. Re-investigating determinants of choice for oral antipsychotics is warranted after introduction of a short-acting parenteral formulation of atypical antipsychotics.

\section{References}

1. Davis JM, Janicak PG, Wang Z, Gibbons RD, Sharma RP (1992) The efficacy of psychotropic drugs: implications for power analysis. Psychopharmacol Bull 28:151-155

2. American Psychiatric Association (1997) Practice guideline for the treatment of patients with schizophrenia. Am J Psychiatry 154[Suppl 4]:1-63

3. Moller HJ (1998) Novel antipsychotics and negative symptoms. Int Clin Psychopharmacol 13[Suppl 3]:S43-S47

4. Leucht S, Pitschel-Walz G, Abraham D, Kissling W (1999) Efficacy and extrapyramidal side-effects of the new antipsychotics olanzapine, quetiapine, risperidone, and sertindole compared to conventional antipsychotics and placebo. A metaanalysis of randomized controlled trials. Schizophr Res 35:51-68

5. Joy CB, Adams CE, Lawrie SM (2001) Haloperidol versus placebo for schizophrenia (Cochrane Review). Cochrane Database Syst Rev 2:CD003082
6. Sarfati Y, Olivier V, Bouhassira M (1999) New antipsychotics in the treatment of schizophrenia. A European survey (in French). Encephale 25:658-666

7. Duggan L, Fenton M, Dardennes RM, El-Dosoky A, Indran S (2002) Olanzapine for schizophrenia (Cochrane Review). In: The Cochrane Library Issue no. 4. Update Software, Oxford

8. Geddes J, Freemantle N, Harrison P, Bebbington P (2000) Atypical antipsychotics in the treatment of schizophrenia: systematic overview and meta-regression analysis. BMJ 321:13711376

9. Tuunainen A, Wahlbeck K, Gilbody SM (2002) Newer atypical antipsychotic medication versus clozapine for schizophrenia (Cochrane Review). In: The Cochrane Library, Issue no. 4. Update Software, Oxford

10. Wahlbeck K, Cheine M, Essali MA (2002) Clozapine versus typical neuroleptic medication for schizophrenia (Cochrane Review). In: The Cochrane Library, Issue no. 4. Update Software, Oxford

11. Wahlbeck K, Cheine M, Tuisku K, Ahokas A, Joffe G, Rimon R (2000) Risperidone versus clozapine in treatment-resistant schizophrenia: a randomized pilot study. Prog Neuropsychopharmacol Biol Psychiatry 24:911-922

12. Srisurapanont M, Disayavanish C, Taimkaew K (2002) Quetiapine for schizophrenia (Cochrane Review). In: The Cochrane Library, Issue no. 4. Update Software, Oxford

13. Kennedy E, Song F, Hunter R, Clarke A, Gilbody S (2002) Risperidone versus typical antipsychotic medication for schizophrenia (Cochrane Review). In: The Cochrane Library, Issue no. 4. Update Software, Oxford

14. Meyer JM (2002) A retrospective comparison of weight, lipid, and glucose changes between risperidone- and olanzapinetreated inpatients: metabolic outcomes after 1 year. J Clin Psychiatry 63:425-433

15. Buitelaar JK, Ewijk WMv, Harms HH, Kahn RS, Linszen DH, Loonen AJM, Louwerens JW, Sloof CJAJ (1998) Guideline for the treatment with antipsychotics in schizophrenic psychosis (in Dutch). Dutch Psychiatric Association. Boom, Amsterdam

16. Herings RM, Bakker A, Stricker BH, Nap G (1992) Pharmacomorbidity linkage: a feasibility study comparing morbidity in two pharmacy based exposure cohorts. J Epidemiol Community Health 46:136-140

17. American Psychiatric Association (1994) Diagnostic and statistical manual for mental disorders, 4th edn. DSM-IV. American Psychiatric Association, Washington, D.C.

18. Anonymous (2000) Anatomical Therapeutical Chemical (ATC) classification index. WHO Collaboration Centre for Drugs Statistics Methodology (Oslo)

19. Fenton M, Coutinho ES, Campbell C (2001) Zuclopenthixol acetate in the treatment of acute schizophrenia and similar serious mental illnesses (Cochrane Review). Cochrane Database Syst Rev 3:CD000525

20. Rosenheck R, Evans D, Herz L, Cramer J, Xu W, Thomas J, Henderson W, Charney D (1999) How long to wait for a response to clozapine: a comparison of time course of response to clozapine and conventional antipsychotic medication in refractory schizophrenia. Schizophr Bull 25:709-719

21. Ravelli DP, Nolen WA, Knoppert-van der Klein EAM, Bouvy PF, Honig A, Klompenhouwer JL (1998) Guideline pharmacotherapy bipolaire disorders (in Dutch). Dutch Psychiatric Association. Boom, Amsterdam 